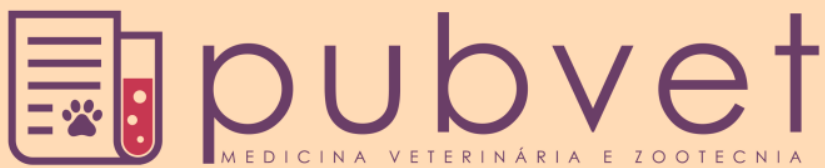

HTTP://DX.DOI.ORG/10.22256/PUBVET,V11N8.819-824

\section{Desenvolvimento inicial de brotações com uso de $1 / 2$ e $1 / 6$ do cladódio na propagação da palma forrageira Nopalea cochenillifera var. miúda}

\author{
José Maurício Maciel Cavalcante ${ }^{1 *}$, Ana Luiza Bleasby Queiroz ${ }^{2}$, Carolaine Cavalcante \\ Oliveira $^{2}$, José Flávio Celestino Sousa Saraiva²
}

${ }^{I}$ Professor do Instituto Federal de Educação Ciência e Tecnologia do Piauí - Campus Uruçuí, Uruçuí, Piauí, Brasil, E-mail: mauricio.cavalcante@ifpi.edu.br

${ }^{2}$ Discentes do curso de Engenharia Agronômica, Instituto Federal de Educação Ciência e Tecnologia do Piauí - Campus Uruçuí, Uruçuí, Piauí, Brasil.

*Autor para correspondência

RESUMO. Este trabalho avaliou o efeito de dois tamanhos de fragmentação de cladódios (1/2 e 1/6 do cladódio) no desenvolvimento de novas brotações para propagação de palma forrageira var. miúda (Nopalea cochenillifera, Salm Dyck). Foram utilizados quatro tratamentos: $\mathrm{T}_{1 / 2}$ (cladódio seccionado longitudinalmente e utilizado uma das metades), Tsup (utilização do terço superior da outra metade do cladódio seccionado longitudinalmente), Tmed (utilização do terço médio) e Tinf (utilização do terço inferior), que foram plantados em recipientes de dois litros com solo:esterco bovino (1:1) e mantidos em casa de vegetação. Os tratamentos foram avaliados 45 dias pós-plantio para o percentual de fragmentos viáveis, número de brotações/fragmento, comprimento, largura e área estimada dos cladódios brotados. Todos os fragmentos do tratamento $\mathrm{T}_{1 / 2}$ permaneceram viáveis contra $73,3 \%$ nos demais tratamentos. Todos os fragmentos viáveis dos tratamentos adotados apresentaram 01 brotação/fragmento. As brotações obtidas em $T_{1 / 2}$ apresentaram maior desenvolvimento quando comparados aos demais tratamentos tanto para comprimento $(\mathrm{P}=0,002)$, largura $(\mathrm{P}=0,001)$ e área estimada $(\mathrm{P}=0,001)$, não havendo diferenças entre os tratamentos Tsup, Tmed e Tinf. Correlação linear positiva $(\mathrm{P}=0,001)$ foi observada entre o peso dos fragmentos utilizados nos tratamentos com o comprimento $(\mathrm{r}=0,899)$, largura $(\mathrm{r}=0,842)$ e área estimada dos cladódios $(\mathrm{r}=0,910)$. O uso de fragmentos de 1/2 e 1/6 do cladódio mostrou ser viável na propagação de Nopalea cochenillifera var. miúda.

Palavras chave: Cactaceae, forragem, propagação vegetativa

\section{Initial development of buds using 1/2 and 1/6 of cladodes in the propagation of forage palm Nopalea cochenillifera var. miúda}

ABSTRACT: This study evaluated the effect of two fragmentation size of cladodes (1/2 e $1 / 6$ do cladodes) on the development of new buds in the propagation of palm forage var. miúda (Nopalea cochenillifera, Salm Dyck). Four treatments were used: $\mathrm{T}_{1 / 2}$ (used one of the halves of cladodes longitudinally sectioned), Tsup (used the upper third of the other half cladode longitudinally sectioned), Tmed (used the middle third) and Tinf (used the lower third), planted in two liters containers with soil:cattle manure (1:1) and maintained in greenhouse. The treatments were evaluated for the percentage of viable fragments, number of buds/fragment, length, width and estimated area of sprouted cladodes at 45 days post-planting of the fragments. All fragments of $\mathrm{T}_{1 / 2}$ treatment remain viable against $73.3 \%$ of the other treatments. All viable fragments of the treatments used presented 01 bud/fragment. The cladodes obtained in $\mathrm{T}_{1 / 2}$ were higher to the other treatments for length 
$(\mathrm{P}=0,002)$, width $(\mathrm{P}=0,001)$ and estimated area $(\mathrm{P}=0,001)$, but there were no differences between treatments Tsup, Tmed and Tinf. Positive linear correlation $(\mathrm{P}=0,001)$ was observed between the weight of the fragments used in the treatments with the length $(\mathrm{r}=0,899)$, width $(\mathrm{r}=0,842)$ and area estimated the cladodes $(\mathrm{r}=0,910)$. The use of $1 / 2$ and $1 / 6$ of cladodes fragments proved to be feasible in the propagation of Nopalea cochenillifera var. miúda.

Keywords: Cactaceae, forage, vegetative propagation

\section{Desarrollo inicial de brotes con uso de 1/2 y 1/6 del cladodio en la propagación de la palma forrajera Nopalea cochenillifera var. miúda}

RESUMEN. Este trabajo evaluó el efecto de dos tamaños de fragmentación de cladodios (1/2 y $1 / 6$ del cladodio) en el desarrollo de nuevos brotes para la propagación de palma forrajera var. miúda (Nopalea cochenillifera, Salm Dyck). Se utilizaron cuatro tratamientos: $\mathrm{T}_{1 / 2}$ (cladodio seccionado longitudinalmente y utilizado una de las mitades), Tsup (uso del tercio superior de la otra mitad del cladodio seccionado longitudinalmente), Tmed (uso del tercio medio) y Tinf (uso del tercio inferior), que se plantaron en recipientes de dos litros con suelo:estiércol bovino (1:1) y mantenidos en casa de vegetación. Los tratamientos se evaluaron 45 días después de la plantación para el porcentaje de fragmentos viables, número de brotes/fragmento, longitud, anchura y área estimada de los cladodios brotados. Todos los fragmentos del tratamiento $\mathrm{T}_{1 / 2}$ permanecieron viables frente al 73,3\% en los demás tratamientos. Todos los fragmentos viables de los tratamientos adoptados presentaron 01 brotación/fragmento. Las brotes obtenidas en $T_{1 / 2}$ presentaron mayor desarrollo cuando se compararon a los demás tratamientos tanto para longitud $(\mathrm{P}=0,002)$, anchura $(\mathrm{P}=0,001)$ y área estimada $(\mathrm{P}=0,001)$, no habiendo diferencias entre los tratamientos Tsup, Tmed e Tinf. Correlación lineal positiva $(\mathrm{P}=0,001)$ se ha observado entre el peso de los fragmentos utilizados en los tratamientos con la longitud $(r=0,899)$, ancho $(\mathrm{r}=0,842)$ y el área estimada de los cladodios $(\mathrm{r}=0,910)$. El uso de fragmentos de $1 / 2$ y 1/6 del cladodio mostró ser viable en la propagación de Nopalea cochenillifera var. miúda.

Palabras clave: Cactaceae, forraje, propagación vegetativa

\section{Introdução}

A palma forrageira tem sido amplamente difundida no Nordeste brasileiro por aliar alta produção de matéria verde e rusticidade em ambientes de déficit hídrico, sendo importante recurso forrageiro para a produção animal em períodos de estiagem (Galvão Júnior et al., 2014, Leite et al., 2014). A variedade miúda (Nopalea cochenillifera, Salm Dyck) destaca-se pelo elevado potencial produtivo no cultivo adensado (Silva et al., 2014), maiores teores de matéria seca e carboidratos (Andrade et al., 2002), melhor palatabilidade (Santos et al., 2001), resultando em boa degradabilidade ruminal (Teixeira et al., 1999) e maior consumo de matéria seca e ganho de peso quando comparada a outras variedades palma (Santos et al., 2001).

O método tradicional de formação de um palmal é pelo plantio de cladódios inteiros (Galvão Júnior et al., 2014, Neves et al., 2010). Na forma adensada, necessita-se de 40 a 80 mil cladódios para implantação de um hectare de palma forrageira (Silva et al., 2014), quantidade que pode estar indisponível em localidades onde não há palmais visando a produção de cladódios para propagação. Assim, a adoção de técnicas que favoreçam a multiplicação rápida da palma poderá incrementar sua difusão. Dentre estas técnicas, destaca-se a fragmentação do cladódio, que consiste no seu corte em fragmentos menores e o plantio dos mesmos para obtenção de novas mudas (Neves et al., 2010, Silva et al., 2014). Esta técnica de baixo custo possibilita a implantação de palmais para produção de mudas a partir de uma menor quantidade de cladódios (Neves et al., 2010, Silva et al., 2014).

Apesar da técnica do fracionamento de cladódios ser simples e acessível a pequenos produtores interessados na multiplicação de variedades de interesse (Neves et al., 2010, Silva et al., 2014), poucos são os trabalhos que tratam de sua padronização, particularmente na 
comparação entre tamanhos de frações na variedade palma miúda. Deste modo, o presente trabalhado teve como objetivo comparar o uso de $1 / 2$ e $1 / 6$ do cladódio no desenvolvimento inicial de novas brotações de palma miúda.

\section{Material e Métodos}

O experimento foi desenvolvido na Fazenda Experimental do Instituto Federal do Piauí (IFPI), Campus Uruçuí, no município de Uruçuí - PI $\left(07^{\circ} 17^{\prime}\right.$ Sul e $44^{\circ} 30^{\prime}$ ' Oeste), no período de junho e julho de 2016. Para o experimento foram utilizados cladódios de exemplares de Nopalea cochenillifera, variedade palma miúda, selecionados quanto à sanidade (isentos de pragas e/ou doenças), boa conformação e semelhança no tamanho. Os cladódios selecionados foram segmentados de modo a obter os quatro tratamentos experimentais, conforme a seguir: primeiramente, o cladódio foi seccionado longitudinalmente e uma de suas metades utilizada no plantio (tratamento $\mathrm{T}_{1 / 2}$ ). A outra metade sofreu dois cortes transversais de modo a se obter três fragmentos de tamanhos semelhantes e identificados segundo sua posição no cladódio: superior (tratamento Tsup), médio (tratamento Tmed) e inferior (tratamento Tinf) (Figura 1). Os tratamentos representam uso de metade (tratamento $\mathrm{T}_{1 / 2}$ ) em relação ao uso da sexta parte (tratamentos Tsup, Tmed e Tinf) de cladódios na propagação da palma miúda.

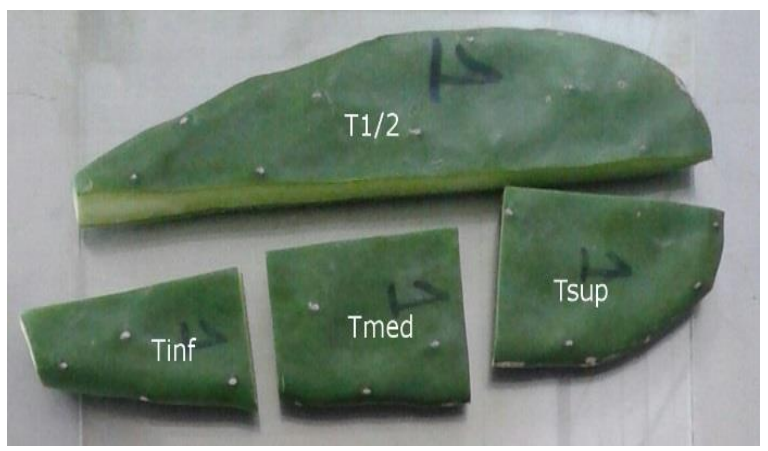

Figura 1. Fragmentação do cladódio inicial nos tratamentos avaliados $\mathrm{T}_{1 / 2}$ (cladódio seccionado longitudinalmente), Tsup (uso da parte superior do cladódio após dois cortes transversais em uma das metades do cladódio), Tmed (uso da parte média) e Tinf (uso da parte inferior).

Cada fragmento foi pesado e quantificado o número de aréolas (gemas) e mantidos à sombra por sete dias para desidratação e cicatrização dos cortes antes do plantio (Neves et al., 2010, Silva et al., 2014). Para o plantio foram utilizados recipientes plásticos com capacidade de dois litros, preenchidos com substrato formado por solo:esterco bovino (1:1). Cada fragmento foi introduzido cuidadosamente no substrato até metade de seu comprimento, de modo a garantir um mínimo de uma aréola em contato com o solo.

Os tratamentos foram alocados em casa de vegetação telada e irrigados duas vezes por semana por 45 dias quando então foram avaliados o percentual os fragmentos viáveis, o número de brotações/fragmento, medição do comprimento e largura dos cladódios brotados, bem como da estimação da área dos cladódios dos brotamentos obtidos. A área do cladódio $\left(\mathrm{AC}, \mathrm{em} \mathrm{cm}^{2}\right)$ foi estimada pela equação $\mathrm{AC}=0,7198 \times \mathrm{CC} \times \mathrm{LC}$, conforme Pinheiro et al. (2015), onde CC e LC são, respectivamente, comprimento e largura (em $\mathrm{cm})$ do cladódio formado aos 45 dias.

O delineamento experimental foi inteiramente casualizado, constituído de quatro tratamentos ( $\mathrm{T}_{1 / 2}$, Tsup, Tmed e Tinf) com cinco repetições. Os dados obtidos nas diferentes variáveis analisadas foram submetidos ao teste de Shapiro - Wilk para avaliação da normalidade dos mesmos. Para a análise das variáveis percentual de fragmentos viáveis e número de brotações foi utilizado o teste do Chi-quadrado. Para as demais variáveis estudadas, as médias obtidas foram comparadas entre os tratamentos com uso da análise de variância, seguidas do teste de Tukey ao nível de $5 \%$ de significância. As variáveis comprimento, largura e área estimada das brotações obtidas foram correlacionadas com o peso de seus respectivos fragmentos por meio da correlação de Pearson. As análises estatísticas foram realizadas com uso do software estatístico IBM SPSS Statistics v.20.

\section{Resultados e Discussão}

Os fragmentos constituintes do tratamento $T_{1 / 2}$ apresentaram peso médio de 79,6 $\pm 19,5 \mathrm{~g}$ e média de aréolas de 30,6 $\pm 4,6$. Os tratamentos Tsup, Tmed e Tinf não diferiram entre si quanto ao peso dos fragmentos utilizados $(26,6 \pm 5,7 \mathrm{~g}, 32,5 \pm 6,2$ $\mathrm{g}$ e $26,1 \pm 6,2 \mathrm{~g}$ respectivamente; $\mathrm{P}=0,22$ ). Entretanto, o tratamento Tsup apresentou quantidade média de aréolas (13,2 $\pm 2,3$ aréolas) significativamente superior aos tratamentos Tmed e Tinf $(8,2 \pm 1,8$ e $8,4 \pm 1,5$ aréolas, respectivamente; $\mathrm{P}=0,002$ ), estando condizente com o fato de que nesta região há uma quantidade maior de aréolas que darão origem a novos cladódios durante o desenvolvimento vegetativo da palma (Reyes-Agüero and Valiente-Banuet, 2006). 
Após 45 dias de plantio, todos os fragmentos do tratamento $\mathrm{T}_{1 / 2}$ permaneceram viáveis enquanto $73,3 \%$ dos demais tratamentos mantiveram-se viáveis no período, sem diferença estatística entre os tratamentos $(\mathrm{P}=0,475)$. Redução da taxa de viabilidade com o aumento do fracionamento de cladódios tem sido observada em Opuntia ficus indica (Solano and Orihuela, 2008) e as perdas observadas no presente trabalho foram registradas nos primeiros 15 dias pósplantio e se caracterizaram por podridão de coloração acastanhada a preta, acometendo principalmente o parênquima do fragmento afetado, lesões compatíveis com os de ataque de fungos e bactérias oportunistas que comumente afetam a palma forrageira (Souza et al., 2010).

Quanto ao número de brotações aos 45 dias de plantio, todos os fragmentos viáveis dos tratamentos apresentaram 01 brotação/fragmento, não havendo diferença entre os tratamentos. A presença do maior número de aréolas ativas em cladódios inteiros pode resultar na formação de um maior número de brotações quando comparado ao uso de fragmentos de cladódios (Singh and Singh, 2003). Entretanto, no presente trabalho, apesar dos diferentes tipos de fragmentação ou da região do cladódio onde estes se localizavam terem apresentado diferenças no número médio de aréolas, isto não resultou num maior número de brotações no período avaliado, sugerindo que, nas condições do presente trabalho, os fragmentos utilizados apresentaram mesmo potencial de surgimento em brotações.

Quanto ao desenvolvimento das novas brotações, este foi influenciado pelo tratamento, conforme observado na Tabela 1 . Os cladódios obtidos em $\mathrm{T}_{1 / 2}$ foram superiores aos demais tratamentos tanto quanto ao comprimento $(\mathrm{P}=$ $0,002)$, largura $(\mathrm{P}=0,001)$ e área estimada $(\mathrm{P}=$ 0,001 ) (Figura 2). Um menor desenvolvimento de cladódios brotados com a redução do tamanho dos fragmentos plantados em Opuntia ficus-indica tem sido observado (Solano and Orihuela, 2008) e atribuído à maior reserva de nutrientes nos fragmentos de cladódio de maior tamanho para a promoção de desenvolvimento de novos cladódios (Singh e Singh, 2003). É possível que o maior peso dos fragmentos do tratamento $\mathrm{T}_{1 / 2}$ implique em maior quantidade de reservas, favorecendo o desenvolvimento de suas brotações quando comparado aos demais tratamentos.

Ainda conforme a Tabela 1, não foram encontradas diferenças entre os tratamentos Tsup,
Tmed e Tinf para comprimento $(\mathrm{P}=0,848)$, largura $(\mathrm{P}=0,663)$ e área $(\mathrm{P}=0,642)$ dos cladódios brotados nestes tratamentos. Diferenças na organogenese de fragme tos obtidos de diferentes regiões do cladódio de cactáceas tem sido observada, a exemplo de um maior desenvolvimento radicular de fragmentos basais de cladódios de Opuntia ficus-indica (StambouliEssassi et al., 2015), o que poderia sugerir uma possível diferença no desenvolvimento de novas brotações em fragmentos conforme a sua localização no cladódio inicial.

Tabela 1. Comprimento $(\mathrm{cm})$, largura e área estimada dos cladódios originado das brotações dos tratamentos $T_{1 / 2}$, Tsup, Tmed e Tinf, após 45 dias de plantio

\begin{tabular}{lccc}
\hline Tratamento & $\begin{array}{c}\text { Comprimento } \\
(\mathrm{cm})\end{array}$ & $\begin{array}{c}\text { Largura } \\
(\mathrm{cm})\end{array}$ & $\begin{array}{c}\text { Área estimada } \\
\left(\mathrm{cm}^{2}\right)\end{array}$ \\
\hline $\mathrm{T}_{1 / 2}$ & $17,8 \pm 4,4 \mathrm{a}$ & $4,7 \pm 0,9 \mathrm{a}$ & $61,2 \pm 21,2 \mathrm{a}$ \\
Tsup & $9,0 \pm 3,2 \mathrm{~b}$ & $2,3 \pm 0,6 \mathrm{~b}$ & $15,7 \pm 9,6 \mathrm{~b}$ \\
Tmed & $8,0 \pm 2,2 \mathrm{~b}$ & $2,0 \pm 0,1 \mathrm{~b}$ & $11,6 \pm 2,9 \mathrm{~b}$ \\
Tinf & $8,6 \pm 1,4 \mathrm{~b}$ & $2,0 \pm 0,2 \mathrm{~b}$ & $12,4 \pm 2,2 \mathrm{~b}$ \\
\hline
\end{tabular}

Médias seguidas de mesma letra numa mesma coluna não diferem entre si $(\mathrm{P}>0,05)$.

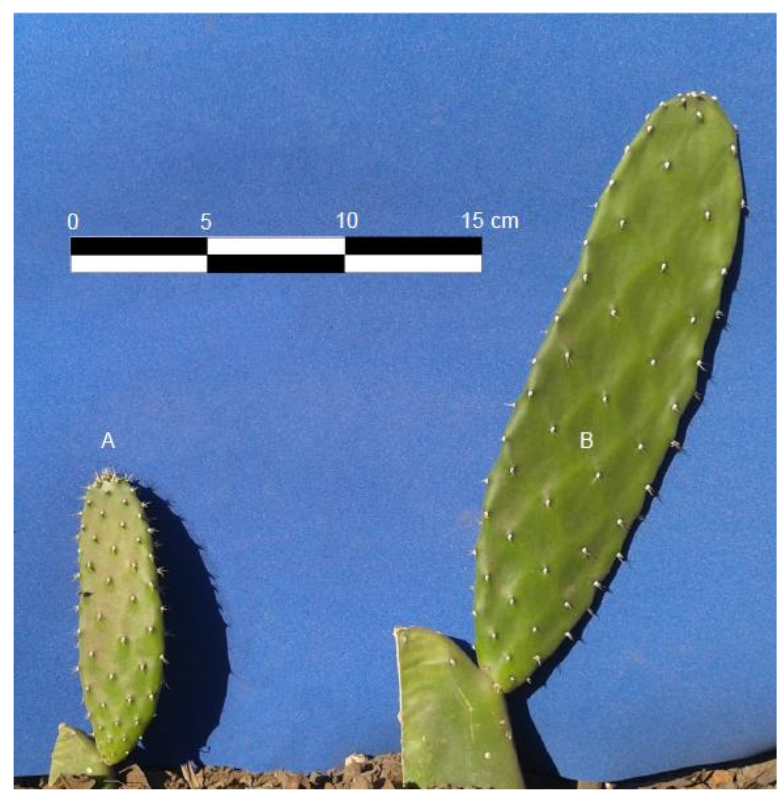

Figura 2. Desenvolvimento de cladódios após 45 dias de plantio dos fragmentos do tratamento Tsup (A) e $\mathrm{T}_{1 / 2}$ (B).

Lima et al. (2013) encontraram maior percentual de brotação de fragmentos obtidos do terço basal de cladódios de Nopalea cochenillifera, var. baiana. Entretanto, estes autores atribuíram esta constatação ao fato de que esta região apresenta maior quantidade de fitomassa no cladódio e, consequentemente, maior quantidade de reservas. No presente trabalho, o peso médio dos tratamentos Tsup, Tmed e Tinf não diferirem estatisticamente entre si e, 
consequentemente, não diferiram na quantidade de reservas para o desenvolvimento de novos cladódios. Assim, não parece haver influência da região do cladódio na quantidade e desenvolvimento das brotações na palma miúda.

A influência do peso dos fragmentos utilizados nos diferentes tratamentos experimentais no desenvolvimento das brotações também pode ser observada na Figura 3. Correlação linear positiva foi observada entre o peso dos fragmentos utilizados nos tratamentos com o comprimento (Figura 3a), largura (Figura 3b) e área estimada (Figura 3c) das brotações $(\mathrm{P}=0,001)$, com coeficientes de correlação (r) de 0,899, 0,842 e 0,910 respectivamente, indicando forte correlação entre as variáveis envolvidas. Por estes resultados, é razoável supor que o uso de fragmentos de cladódio menores que os utilizados neste trabalho poderão implicar num menor desenvolvimento de suas brotações, o que delongaria o processo de obtenção de cladódios maduros para propagação e implantação de palmais desta variedade.

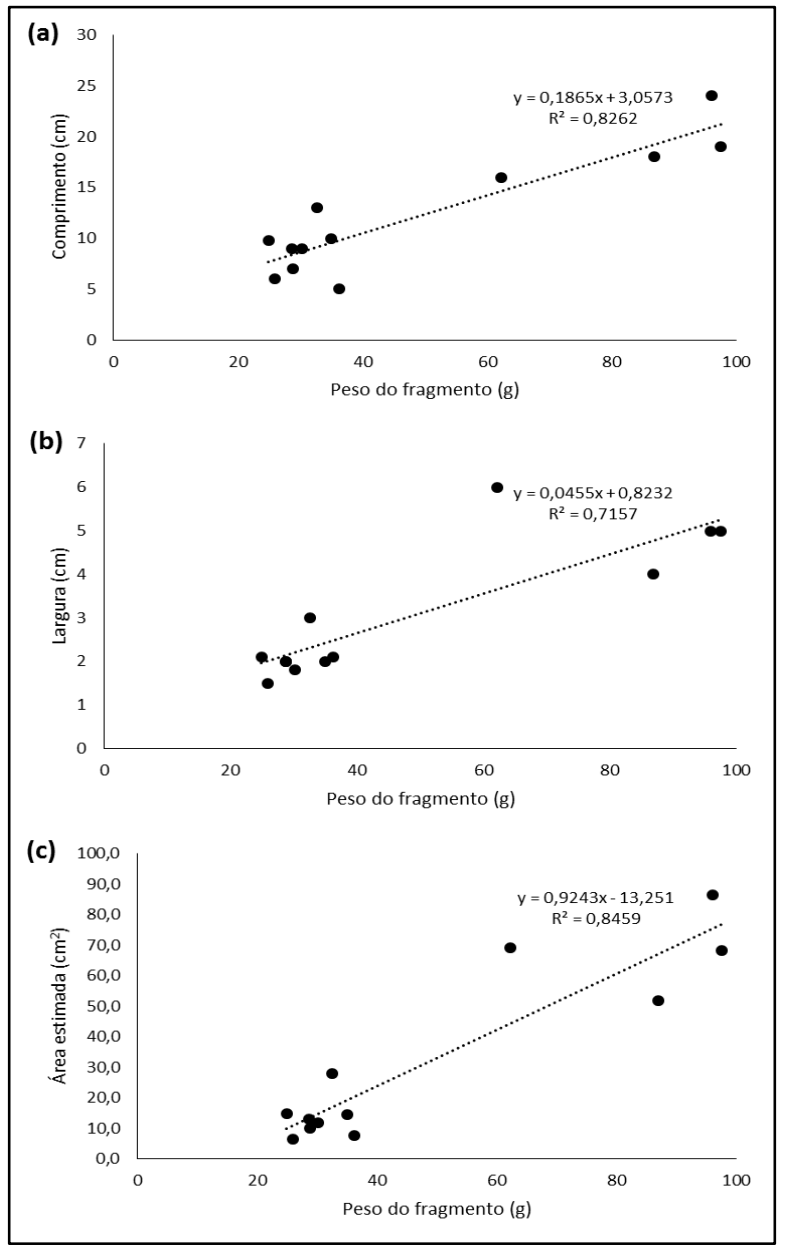

Figura 3. Correlação entre comprimento (a), largura (b) e área estimada (c) de cladódios resultantes das brotações e o peso dos fragmentos dos tratamentos experimentais.

\section{Conclusões}

$\mathrm{O}$ uso de ambas as frações de cladódio avaliadas (1/2 e 1/6 do cladódio) mostrou-se viável na propagação de Nopalea cochenillifera var. miúda. Os tratamentos avaliados apresentam semelhante potencial em gerar novas brotações, independente do tamanho dos fragmentos ou de sua localização no cladódio de origem. Entretanto, tratamentos referentes a fragmentos de $1 / 6$ do cladódio apresentam menor desenvolvimento inicial de suas brotações aos 45 dias pós-plantio em comparação com fragmentos de 1/2 cladódio, possivelmente relacionado ao menor conteúdo de reservas destes fragmentos para suprir 0 desenvolvimento de suas brotações.

\section{Referências Bibliográficas}

Andrade, D. K. B., Ferreira, M., Véras, A. S. C., Wanderley, W. L., Silva, L., Carvalho, F., Alves, K. S. \& Melo, W. 2002. Digestibilidade e absorção aparentes em vacas da raça Holandesa alimentadas com palma forrageira (Opuntia ficus-indica Mill) em substituição à silagem de sorgo (Sorghum bicolor (L.) Moench). Revista Brasileira de Zootecnia, 31, 2088-2097.

Galvão Júnior, J. G. B., Silva, J. B. A., Morais, J. H. G. \& Lima, R. N. 2014. Palma forrageira na alimentação de ruminantes: cultivo e utilização. Acta Veterinaria Brasilica, 8, 7885.

Leite, M. L. M. V., Silva, D. S., Andrade, A. P., Pereira, W. E. \& Ramos, J. P. F. 2014. Caracterização da produção de palma forrageira no Cariri paraibano. Revista Caatinga, 27, 192-200.

Lima, W. B., Pereira, D. D., Nascimento, A. R. L., Albuquerque, A. G. \& Vieira, M. F. 2013. Propagação por fracionamento do cladódio de palmas forrageiras variedade baiana (Nopalea cochenillifera SALM-DYCK). In: SIMPÓSIO BRASILEIRO DE RECURSOS NATURAIS DO SEMIÁRIDO, 2013, Iguatu, CE. Anais...

Neves, A. L. A., Pereira, L. G. R., Santos, R. D., Voltolini, T. V., Araújo, G. G. L., Moraes, S. A., Aragão, A. S. L. \& Costa, C. T. F. 2010. Plantio e uso da palma forrageira na alimentação de bovinos leiteiros no semiárido brasileiro. In: Leite, G. d. (ed.). EMBRAPA, Juiz de Fora.

Pinheiro, K. M., Silva, T. G. F., Diniz, W. J. S., Carvalho, H. F. S. \& Moura, M. S. B. 2015. Indirect methods for determining the area 
index of forage cactus cladodes. Pesquisa Agropecuária Tropical, 45, 163-171.

Reyes-Agüero, J. A. \& Valiente-Banuet, A. 2006. Reproductive biology of Opuntia: a review. Journal of Arid Environments, 64, 549-585.

Santos, D. C., Santos, M. V. F., Farias, I., Dias, F. M. \& Andrade Lira, M. 2001. Desempenho produtivo de vacas 5/8 Holando/Zebu alimentadas com diferentes cultivares de palma forrageira (Opuntia eNopalea). Revista Brasileira de Zootecnia, 30, 12-17.

Silva, L. M., Fagundes, J. L., Viegas, P. A. A., Moreira, A. L. \& Backes, A. A. 2014. Produtividade da palma forrageira cultivada em diferentes densidades de plantio. Ciência Rural, 44, 2064-2071.

Singh, R. S. \& Singh, V. 2003. Growth and development influenced by size, age, and planting methods of cladodes in cactus pear (Opuntia ficus-indica (L.) Mill.). Journal of the Professional Association for Cactus Development, 5, 47-54.

Solano, J. \& Orihuela, A. 2008. Supervivencia y producción de nopal para verdura (Opuntia ficus-indica) utilizando fracciones mínimas. Journal of the Professional Association for Cactus Development, 10, 198-208.

Souza, A. E. F., Nascimento, L. C., Araújo, E., Lopes, E. B. \& Souto, F. M. 2010. Ocorrência e identificação dos agentes etiológicos de doenças em palma forrageira (Opuntia ficusindica Mill.) no semiárido paraibano. Biotemas, 23, 11-20.

Stambouli-Essassi, S., Harrabi, R., Bouzid, S. \& Harzallah-Skhiri, F. 2015. Evaluation of the efficiency of Opuntia ficus-indica cladode cuttings for vegetative multiplication. Notulae Botanicae Horti Agrobotanic, 43, 521-527.

Teixeira, J. C., Evangelista, A. R., Perez, J. R. O., Trindade, I. A. C. \& Moron, I. R. 1999. Cinética da digestão ruminal da palma forrageira (nopalea cochenillifera (1.) lyonscactaceae) em bovinos e caprinos. Ciência Agrotecnologia, 23, 179-186.

\section{Article History:}

Received 16 April 2017

Accepted 16 May 2017

Available on line 17 July 2017

License information: This is an open-access article distributed under the terms of the Creative Commons Attribution License 4.0, which permits unrestricted use, distribution, and reproduction in any medium, provided the original work is properly cited. 